\title{
Mudanças oculares durante a gestação
}

\author{
Ocular changes during pregnancy
}

\author{
Enéias Bezerra Gouveia ${ }^{1}$ \\ Paulo Sergio Pereira Conceição ${ }^{2}$ \\ Maira Saad de Avila Morales ${ }^{3}$
}

\begin{tabular}{|l|}
\hline RESUMO \\
\hline A gestação pode causar várias alterações no funcionamento do olho \\
humano, tanto nas condições sistêmicas quanto em doenças oculares, \\
sendo seus efeitos divididos em três categorias: alterações fisiológicas, \\
mudanças em doenças oculares pré-existentes e alterações patológicas. O \\
presente texto propõe uma revisão bibliográfica sobre o tema. Os autores \\
pesquisaram os bancos de dados do PubMed (MEDLINE) e LILACS.
\end{tabular}

Descritores: Complicações na gravidez; Gravidez/fisiologia; Doenças da coróide; Doenças retinianas; Retinopatia diabética; Soluções oftálmicas; Pré-eclâmpsia

\section{INTRODUÇÃO}

A gravidez está associada a diversas mudanças envolvendo múltiplos órgãos, incluindo os olhos. Os efeitos da gravidez sobre os olhos podem ser divididios em três categorias: alterações fisiológicas, mudanças em doenças oculares pré-existentes e alterações patológicas.

As alterações fisiológicas oculares mostram-se bem documentadas em relação à córnea, pressão intraocular e acomodação.

A córnea apresenta diminuição em sua sensibilidade, aumento de sua espessura e curvatura que causam mudanças temporárias na refração, bem como intolerância ao uso de lentes de contato. Há uma diminuição da pressão intraocular, principalmente no terceiro trimestre de gravidez, decorrente de aumento do escoamento do humor aquoso pela via tradicional ou uveoescleral e, também, pela redução da produção do humor aquoso provocado pela acidose proveniente das alterações hormonais ${ }^{(1)}$. A literatura descreve uma perda transitória da acomodação durante e após a gravidez ${ }^{(2)}$, e também insuficiência e paralisia de acomodação são registradas em associação à lactação( ${ }^{(2)}$.

A gravidez pode alterar o curso de doenças oculares pré-existentes, como uveíte, retinopatia diabética, etc.

Da mesma forma pode causar condições específicas, como cegueira cortical associada à pré-eclâmpsia/eclâmpsia, ou pode contribuir no desenvolvimento de doenças que também ocorrem em pacientes não grávidas, como a retinopatia serosa central.

\section{EFEITOS SISTÊMICOS DA GRAVIDEZ}

A maioria das mudanças oculares durante a gravidez que afetam os olhos tem natureza hormonal, metabólica, hematológica, cardiovascular e imunológica.

Dentre as muitas mudanças na produção de hormônios maternos, dá-se a redução do hormônio do crescimento e aumento dos níveis da prolactina. A concentração sérica da tiroxina encontra-se elevada, bem como a sua 
proteína carreadora, de modo que a sua fração livre não aumenta significativamente. Os níveis de calcitoninas, aldosterona, renina, angiotensina I e II, cortisol e testoterona apresentam-se todos elevados. Quanto às mudanças metabólicas, destacam-se o ganho de peso pela retenção hídrica, havendo uma redução na osmolalidade. Muitas pacientes apresentam alcalose respiratória por hiperventilação. A gravidez é considerada potencialmente diabetogênica devido ao aumento da atividade e dos níveis de estrógeno, progesterona e cortisol.

As mudanças hematológicas são decorrentes do aumento do volume sanguíneo que chega aumentar aproximadamente $45 \%$, com ganhos de eritrócitos de $33 \%$, havendo ainda um aumento no nível dos fatores e ativadores da coagulação.

Em relação às alterações cardíacas podemos citar: aumento do pulso e da sobrecarga cardíaca, diminuição da pressão arterial e resistência periférica.

A gravidez é considerada como um estado de imunossupressão relativa, com diminuição tanto da imunidade celular ${ }^{(3)}$ como humoral, que desestabilizam várias doenças imunológicas durante a gestação como lupus eritematoso sistêmico e doença de Graves ${ }^{(4)}$.

\section{MUDANÇAS OCULARES "FISIOLÓGICAS"}

\section{A - Glaucoma}

A pressão intraocular (PIO) diminui durante a gravidez ${ }^{(5-8)}$ e, aproximadamente $10 \%$ das gestantes com olhos saudáveis apresentam diminuição da $\mathrm{PIO}^{(9)}$. Este fato é registrado, principalmente, durante a segunda metade da gravidez ${ }^{(1,8)}$, e tende a persistir por vários meses pós-parto ${ }^{(8)}$.

Ainda é desconhecido o mecanismo de a gravidez alterar a PIO em pacientes glaucomatosas. Contudo, o aumento da drenagem do humor aquoso através da via uveoescleral é uma das possíveis razões para esta diminuição ${ }^{(6,10)}$, que seria decorrente do aumento da progesterona ${ }^{(6)}$ e da relaxina ${ }^{(10)}$, que bloqueariam o efeito hipertensivo ocular dos corticóides endó$\operatorname{genos}^{(4)}$. E ainda ocorreria diminuição da pressão venosa episcleral como resultante da diminuição da pressão venosa em sua extremidade superior ${ }^{(11)}$. Uma outra razão proposta para a redução da PIO é a acidose gravídica ${ }^{(12)}$.

Já foi descrito melhora do glaucoma pré-existente durante a gravidez, sendo raro o aparecimento do quadro glaucomatoso durante o período gestacional ${ }^{(12)}$.

\section{B - Córnea}

A sensibilidade corneana encontra-se diminuída na maioria das gestantes ${ }^{(13-14)}$, com redução mais expressiva no final da gravidez. Seis a oito semanas pós-parto, a sensibilidade está normal ${ }^{(13)}$. O aumento da espessura da córnea é mínino e é decorrente de edema ${ }^{(13)}$. No entanto, a quantidade de espessamento não está correlacionada à redução da sensibilidade.
Fuso de Krukemberg pode ser observado em grávidas, mas tende a diminuir de tamanho ao longo do terceiro trimestre até o pós-parto ${ }^{(15)}$. Acredita-se que o aumento desta pigmentação seja decorrente das alterações hormonais e o aumento da dinâmica do humor aquoso seja responsável pelo clareamento do pigmento no final da gravidez ${ }^{(15)}$.

\section{1) Cirurgia refrativa}

Embora existam poucas publicações sobre o resultado de cirurgia refrativa em grávidas antes, durante ou após o parto, a gravidez é considerada pela maioria dos pesquisadores uma contraindicação para ceratotomia fotorrefrativa (PRK) e laser in situ keratomileusis (Lasik) ${ }^{(16)}$.

\section{2) Intolerância à lente de contato}

Nos casos de mulheres com sucesso no uso prévio de lente de contato, muitas delas, quando grávidas, passam a desenvolver intolerância ${ }^{(9,13,17)}$. Isto deve estar relacionado ao aumento da espessura e curvatura corneana ${ }^{(9,13)}$, bem como a alterações na composição da lágrima ${ }^{(9,13)}$. Por este motivo, recomenda-se algumas semanas pós-parto para prescrever uma nova correção ${ }^{(9,13)}$.

\section{DOENÇAS PRÉ-EXISTENTES}

\section{A - Retinopatia diabética}

A gravidez é considerada fator de risco independente para progressão de retinopatia diabética ${ }^{(18)}$. Outros fatores que causam a aceleração de retinopatia diabética neste grupo inclui duração do diabetes ${ }^{(19)}$, hipertensão associada à gravidez e pré-eclâmpsia ${ }^{(20)}$, normalização rápida dos níveis de glicose durante a gravidez ${ }^{(21)}$, controle glicêmico não rigoroso prégravídico $^{(19)}$ e mudança no fluxo sanguíneo retiniano ${ }^{(22)}$. Diabetes gestacional, na ausência de diabetes pré-existente, não mostra uma associação similar com retinopatia diabética.

Existe ainda uma associação no aumento do risco de perda fetal e outras complicações obstétricas ${ }^{(23-25)}$. Estudos de longo período sugerem que a retinopatia não aparenta ser mais grave em pacientes nulíparas que nas multíparas ${ }^{(26-27)}$, portanto o número de partos não eleva o risco de piora da retinopatia ${ }^{(28)}$, sendo que a retinopatia é menos grave em mulheres com duas ou mais gestações ${ }^{(28)}$.

A progressão da retinopatia durante a gravidez é muito influenciada pela coexistência de hipertensão e pré-eclâmpsia ${ }^{(18,29)}$, e está diretamente relacionada com a gravidade da retinopatia pré-existente e, ainda, fortemente influenciada pela duração da diabetes antes da concepção ${ }^{(30-31)}$. Vários estudos mostram que a piora da retinopatia está associada com o baixo controle glicêmico antes da concepção e durante a gravidez ${ }^{(23,25-26)}$.

De acordo com o estudo de diabetes no início da gestação ${ }^{(19)}, 10,3 \%$ das mulheres sem retinopatia e $21,1 \%$ das mulheres com microaneurismas, porém sem outra retinopatia, apresentam progressão da doença durante ou após a gravidez. $\mathrm{Na}$ retinopatia não proliferativa leve houve progressão em $18,8 \%$ das mulheres diabéticas $(6,3 \%$ da retinopatia diabética 
proliferativa), no entanto retinopatia diabética não proliferativa moderada progrediram em $54,8 \%$ dos casos (29\% da retinopatia diabética proliferativa). Os casos de retinopatia diabética proliferativa que evoluiram com complicações que necessitaram de tratamento cirúrgico, tais como descolamento de retina regmatogênico e tracional, e glaucoma neovascular, estão associados com um pior resultado visual ${ }^{(32)}$.

O controle glicêmico durante a gravidez está associado à diminuição no risco de macrossomia fetal e malformação congênita ${ }^{(33-34)}$. Por sua vez, o controle da glicemia antes da gravidez, por um período longo, melhora o curso da retinopatia ${ }^{(35)}$.

Como nas mulheres diabéticas não grávidas, é comum ocorrer uma piora transitória da retinopatia diabética quando temos um controle rápido da glicemia no início da gestação ${ }^{(29)}$.

$\mathrm{O}$ tratamento das gestantes com retinopatia diabética depende da gravidade da doença na ocasião da gestação. Mulheres sem evidências de retinopatia ou com microaneurismas, somente deveriam ser avaliadas durante o primeiro trimestre e, outra vez, quando houver queixas visuais, tais como "floaters", embaçamemto visual ou perda de visão. A avaliação de pacientes com retinopatia diabética não proliferativa inclui exame fundoscópico e retinografia no primeiro trimestre. Aquelas com retinopatia leve devem ser reavaliadas durante o segundo trimestre e mensalmente no terceiro. Pacientes com retinopatia diabética não proliferativa moderada deveriam ser examinadas de quatro em quatro semanas. Normalmente, a progressão da retinopatia piora no final do segundo trimestre ${ }^{(36)}$.

As recomendações atuais para o tratamento incluem: fotocoagulação em gestantes com retinopatia diabética não proliferativa grave. Nesse caso, aguarda-se o estágio proliferativo até apresentar complicações que requeiram cirurgia vitreorretiniana ${ }^{(32)}$, que tecnicamente é mais difícil em grávidas devido às repercusões sistêmicas e fetais. Retinopatia diabética proliferativa requer pan-fotocoagulação para diminuir sua progressão.

A conduta mais importante é a prevenção. $O$ ideal seria a paciente ter um bom controle glicêmico e a retinopatia ser tratada previamente à concepção ${ }^{(37)}$.

A regressão da retinopatia diabética é comum durante o puerpério ${ }^{(38)}$. Apesar disto, mulheres apresentam risco de progressão por até um ano após o parto e podem ter complicações como hemorragia vítrea, descolamento de retina se não tratadas ${ }^{(38)}$. A monitorização deve continuar no período pós-parto para prevenir estas complicações.

É concenso para o planejamento das grávidas diabéticas: (1) controle da glicemia durante toda a gravidez para o bem estar da mãe e do feto ${ }^{(39)}$; (2) é indicado nas diabéticas engravidar o mais precoce possível, uma vez que o período da diabetes é o primeiro fator de risco para a presença, gravidade e progressão da retinopatia nas diabéticas grávidas ${ }^{(23)}$; (3) todas as gestantes diabéticas devem realizar exame oftalmológico no primeiro trimestre.

\section{B - Uveítes}

O impacto da gravidez no curso das doenças inflamatórias não está bem estabelecida, embora haja relatos na literatura da ocorrência de exacerbação de doença. Nenhum desses estudos, contudo, examinaram os resultados da visão em pacientes com uveítes que tenham engravidado.

Rabiah e Vitale ${ }^{(40)}$ examinaram o curso de uveítes não infecciosa durante a gravidez e no período pós-parto, incluindo pacientes com doença de Behçet, Vogt-KoyanagiHarada (VKH) e uveíte idiopática. Quanto ao número de exacerbações da uveíte, $58 \%$ das grávidas tiveram uma, $14 \%$ tiveram duas, e $28 \%$ não apresentaram nenhuma exacerbação do quadro.

Alguns relatos de casos têm sido mencionados na literatura, como a progressão de fibrose sub-retiniana três meses após o parto em pacientes com coroidite multifocal idiopática $^{(41)}$. Em um estudo de artrite reumatóide juvenil idiopática, nenhum dos pacientes com iridociclite crônica tiveram exacerbação durante a gravidez, contudo $6 \%$ destes pacientes apresentaram "flare" um ano após o parto ${ }^{(42)}$. A série de Rabiah e Vitale mostrou que $85 \%$ das grávidas com VKH foi associado com "flare" durante a gravidez, principalmente nos quatro primeiros meses ${ }^{(40)}$.

Um recente estudo em quatro mulheres com uveíte crônica revelou que a atividade inflamatória foi pior no primeiro trimestre e nos três meses pós-parto ${ }^{(43)}$.

Toxoplasmose congênita é o resultado de infecção materna ocorrida durante a gravidez, portanto, não se deveria esperar que o feto de uma gestante com retinocoroidite ativa ou cicatrizada tivesse risco de contrair toxoplasmose congênita na gestação ${ }^{(44)}$. A presença de altos títulos de hemaglutinação não aparenta ter efeito adverso na gravidez, mas não se tem informações quanto aos riscos de aumento do título durante a gravidez $^{(45)}$.

De um modo geral, mulheres, em idade fértil, com sorologia negativa para toxoplasmose devem ser orientadas no pré-natal sobre o risco de adquirir a doença no primeiro trimestre ${ }^{(44)}$.

\section{C - Doença de Graves}

A doença de Graves pode agravar-se no início da gravidez, havendo melhora a partir da metade da gestação, com recorrência no período pós-parto ${ }^{(46)}$. Não há diferença entre os sinais oculares entre as mulheres grávidas e não grávidas, tais como exoftalmia, retração da pálpebra superior e sinal de Kocher.

\section{D - Alta miopia}

Estudo realizado com grávidas portadoras de alta miopia ${ }^{(47)}$, concluiu que a manobra de Valsalva, durante o trabalho de parto normal, não resulta em complicações oculares.

\section{E - Sarcoidose}

A gravidez deve exercer um efeito benéfico na sarcoidose, somente apresentando desenvolvimento ou exacerbação de uveíte e retinopatia no período pós-parto(48-49). Isto é atribuído à supressão imunológica relativa e ao alto nível de corticóide durante a gravidez, com um rápido retorno aos níveis não gravídicos. 


\section{MUDANÇAS PATOLÓGICAS}

\section{A - Doença específica da gravidez}

\section{1) Pré-eclâmpsia e eclâmpsia}

A hipertensão induzida na gravidez, embora rara, ainda é uma importante causa de baixa visual durante a gravidez e pós-parto. Sua terminologia tem mudado durante os anos. Denomina-se hipertensão induzida pela gravidez àquela que ocorre durante a gravidez em uma paciente previamente normotensa. Nesta categoria estão a pré-eclâmpsia, um estado de hipertensão onde os níveis sistólicos são iguais ou superiores a $140 \mathrm{mmHg}$ e os diastólicos iguais ou superiores a $90 \mathrm{mmHg}$, acompanhado de edema e proteinúria, geralmente ocorrendo depois da $20^{\underline{a}}$ semana de gestação. A préeclâmpsia é considerada grave quando a pressão arterial é igual ou superior a $160 \mathrm{X} 110 \mathrm{mmHg}$, acrescida de proteinúria elevada, oligúria, edema pulmonar, dor abdominal, disfunção hepática, anormalidades visuais ou cerebrais. Eclâmpsia é caracterizada pelo desenvolvimento de convulsões tônicoclônica em paciente com pré-eclâmpsia. Nos casos de mulheres já hipertensas, que assim se mantém durante a gravidez, denomina-se hipertensão crônica, porém se a hipertensão é agravada na gestação teremos a pré-eclâmpsia ou eclâmpsia sobreposta à hipertensãa ${ }^{(50)}$.

Distúrbios visuais tais como escotoma, fotopsia, diplopia e embaçamento visual, são descritos em 30-50\% dos pacientes com eclâmpsia, e 20-25\% de pacientes com pré-eclâmpsia grave e hipertensão ${ }^{(51)}$.

As queixas visuais devem ser consideradas um sinal iminente de convulsão em pacientes com pré-eclâmpsia ${ }^{(52)}$. Embora encontrem-se anormalidades da conjuntiva, retina, coróide, nervo óptico e córtex visual, o achado mais comum é a constricção arteriolar ${ }^{(50-51)}$, ocorrendo em aproximadamente $60 \%$ dos pacientes com pré-eclâmpsia ${ }^{(50)}$. A constricção pode ser generalizada ou localizada, acompanhada de outros sinais de retinopatia hipertensiva como hemorragias, exsudatos e edema de retina ${ }^{(50)}$.

Descolamento seroso de retina chega a ocorrer em $10 \%$ de pacientes com eclâmpsia e em 1-2\% de pacientes com préeclâmpsia grave $e^{(50,53)}$. Alguns casos de cegueira transitória são atribuidos à isquemia do nervo óptico, vasoespasmo e edema retiniano; no entanto, a maioria dos casos de cegueira associada com pré-eclâmpsia e eclâmpsia são atribuídas às alterações no córtex occipital ${ }^{(54)}$.

O prognóstico de pacientes com sintomas oculares associados à pré-eclâmpsia e eclâmpsia é bom ${ }^{(54)}$, mesmo em casos de edema de retina exsudativo, e alterações do epitélio pigmentado da retina (EPR) $)^{(55)}$.

\section{2) Cegueira cortical}

A cegueira cortical é uma complicação rara da préeclâmpsia/eclâmpsia, ocorrendo em $15 \%$ dos $\operatorname{casos}^{(56)}$, podendo ocorrer antes ou depois do parto ${ }^{(57)}$. Caracteriza-se por perda visual que pode variar de 4 a 192 horas $^{(56)}$, com exame oftalmoscópico normal e pupilas fotorreagentes. A tomografia computadorizada detecta lesões de baixa densidade, geralmente bilateral, em córtex occipital ${ }^{(56-57)}$ e a ressonância nuclear magnética demonstra correspondente lesões de hiper-intensidade ${ }^{(58)}$ na córtex occipital ou na córtex occipital e parietal. Estes achados indicam a presença de edema cerebral, que possivelmente decorre de um vasoespasmo generalizado ou localizado, que causaria uma isquemia transitória, resultando em edema citotóxico; em um segundo momento, teríamos a desrregulação da circulação posterior, resultando em aumento da permeabilidade causando edema vasogênico ${ }^{(59-61)}$.

A cegueira cortical geralmente tem resolução espontânea ${ }^{(57)}$, mas tem registro de escotoma inferior bilateral sintomática e defeito de campo visual assintomática ${ }^{(62)}$ que persistem por vários meses pós-parto.

Outras causas de grave diminuição da acuidade visual durante a gravidez ou imediatamente após a gestação, com ou sem toxemia pré-existente incluem: oclusão de artéria e veia retiniana, trombose venosa cerebral, neuropatia óptica isquêmica e neurite óptica seguida de hemorragia recorrente e hemorragia vítrea ${ }^{(63-64)}$.

\section{B - Doença não específica da gravidez}

\section{1) Coriorretinopatia serosa central}

A coriorretinopatia serosa central (CRSC) é definida como um descolamento seroso idiopático da retina neurosensorial, secundário a um vazamento focal. Geralmente causa uma diminuição da acuidade visual de leve a moderada, acompanhada de sintomas de metamorfopsia, micropsia, alteração da visão das cores. Vinte a trinta por cento dos pacientes tem uma ou mais recorrências no mesmo olho, e um terço deles tem alterações focais no EPR no olho contralateral. A doença pode ser assintomática se a fóvea não for envolvida ou se o episódio ocorrer no olho não dominante.

Embora esta condição seja dez vezes mais comum em homens que em mulheres, tem uma forte associação à gravidez ${ }^{(65)}$ podendo surgir em qualquer um dos três trimestres da gravidez, embora em metade dos casos ocorra no terceiro trimestre, mesmo que esteja evoluindo sem intercorrências ${ }^{(65-66)}$.

As causas propostas são intrínsecas às condições especiais da gravidez que incluem: alterações hormonais e hemodinâmicas, como aumento do volume das células sanguíneas, alterações da permeabilidade vascular com diminuição da pressão osmótica e hipercoagubilidade ${ }^{(67)}$.

Os casos estudados demonstraram, em sua maioria, uma resolução espontânea no final da gestação ou nos primeiros meses após o parto, com a acuidade visual retornando ao seu patamar anterior ou deixando poucas sequelas ${ }^{(68)}$.

\section{2) Melanoma da coróide}

Há vários relatos que durante a gravidez há um rápido crescimento tumoral de melanoma quiescentes ${ }^{(69-70)}$, bem como o surgimento de melanoma de coróide em pacientes, previamente sadias e sem nenhuma doença ocular ${ }^{(69-70)}$, que foram atribuídos ao aumento do hormônio estimulador de melanócitos ${ }^{(69)}$. 


\section{USO DE MEDICAÇÃO OFTALMOLÓGICA DURANTE A GRAVIDEZ}

Muitas medicações são consideradas seguras durante a gravidez apesar da falta de seguimento clínico envolvendo mulheres grávidas. Um recente estudo sugeriu que a maioria das drogas oftalmológicas apresenta baixo risco para a mãe e o desenvolvimento fetal ${ }^{(71)}$.

\section{A - Hipotensores oculares}

Os beta-bloqueadores são considerados teratogênicos ${ }^{(72)}$ e são secretados e concentrados no leite materno, de modo que não são recomendados durante a gravidez e a lactação ${ }^{(73)}$. A acetazolamina está associada à acidose neonatal e catarata. Estudos em ratos demonstraram teratogenicidade em doses dez vezes àquelas recomendadas em humanos ${ }^{(74-75)}$. Os análogos da prostaglandina, como o latanoprost, são drogas na categoria C, baseados em resultados de animais grávidos expostos à alta dose, confirmado em recente estudo, não registrando nenhum efeito adverso para a grávida ou recém-nascido apesar desta exposição ${ }^{(76)}$.

\section{B - Esteróides tópicos}

O uso de corticóide em grávidas é evitado durante toda a gravidez, no entanto, é necessário para preservação visual em alguns casos de uveíte. Esteróides tópicos em animais foram causadores de teratogenicidade ${ }^{(77)}$, embora em humanos não demonstram causar defeitos em fetos.

Como toda medicação deve apenas ser usada durante a gravidez se o benefício previsto justificar o risco potencial para o feto. Recomenda-se ocluir o ponto lacrimal durante a instilação na tentativa de reduzir a absorção sistêmica da droga.

\section{C - Fluoresceína}

A fluoresceina sódica, utilizada na angiofluoresceinografia, deve ser evitada em gestantes, especialmente no primeiro trimestre. Os efeitos sobre a organogênese fetal ainda não são totalmente conhecidos, no entanto, cruza a barreira placentária em humanos, por terem sido encontrados durante amniocentece em paciente que se encontrava na $33^{\underline{a}}$ semana e havia sido submetida a exame de angiografia ${ }^{(78)}$. Assim, o procedimento deve ser realizado quando existir risco elevado de perda importante da função visual.

As drogas que já são consideradas como potencialmente teratogênicas na administração sistêmica, não devem ser utilizadas por nenhuma outra via em gestantes, dentre elas a tetraciclina $^{(79)}$ e agente antivirais para herpes.

\section{CONCLUS ÃO}

A gravidez representa uma ampla variedade de alterações sistêmicas que pode ter uma repercussão ocular. As gestantes diabéticas deveriam realizar exame oftalmológico logo após a concepção, bem como ser estimuladas ao controle glicêmico antes e durante a gravidez. As mulheres portadoras de uveítes, em idade fértil, devem ser orientadas sobre o risco de ativação da doença no primeiro trimestre ou pós-parto, aconselhando um acompanhamento durante esses períodos. Queixas visuais agudas devem ser valorizadas por serem sinais de pré-eclâmpsia grave ou iminência de eclâmpsia. A cirurgia refrativa deve ser postergada até a estabilidade refracional após o parto. Deve evitar-se ainda a prescrição de novas lentes de contato até o parto, devido à intolerância desenvolvida por muitas mulheres nesse período. É fundamental o trabalho conjunto entre oftalmologistas e obstetras na decisão de prescrição de determinadas medicações durtante a gravidez, como também as pacientes deveriam ser orientadas a realizar a compressão do ducto nasolacrimal durante a instilação de colírios para reduzir a absorção.

O desenvolvimento da Medicina e o rigoroso controle clínico no pré-natal têm melhorado o prognóstico das complicações oculares durante a gravidez

\section{ABSTRACT}

Pregnancy can cause several alterations in human eye function in healthy condition as well in ocular disease; these effects on the eyes are divided into three categories: physiologic alterations, ocular diseases changes already existing before the pregnancy, and pathological alterations. The present text proposes a bibliographical revision on the theme. The authors researched PubMed (MEDLINE) and LILACS databases.

Keywords: Pregnancy complications; Pregnancy/physiology; Choroid diseases; Retinal diseases; Diabetic retinopathy; Ophthalmic solutions; Pre-eclampsia

\section{REFERÊNCIAS}

1. Phillips CI, Gore SM. Ocular hypotensive effect of late pregnancy with and without high blood pressure. Br J Ophthalmol. 1985;69(2):117-9.

2. Sédan J. Répercussion de la vie génitale de la femme sur son appareil oculaire. Ann Ocul (Paris). 1967;200(2):163-76

3. Tomoda Y, Fuma M, Miwa T, Saiki N, Ishizuka N. Cell-mediated immunity in pregnant women. Gynecol Invest 1976;7(5):280-92.

4. Froelich CJ, Goodwin JS, Bankhurst AD, Williams RC Jr. Pregnancy, a temporary fetal graft of suppressor cells in autoimmune disease? Am J Med. 1980;69(3):329-31.

5. Avasthi P, Sethi P, Mithal S. Effect of pregnancy and labor on intraocular pressure. Int Surg. 1976;61(2):82-4.

6. Becker B, Friedenwald JS. Clinical aqueous outflow. AMA Arch Ophthalmol. 1953;50(5):557-71.

7. Horven I, Gjonnaess H. Corneal indentation pulse reduction in pregnancy. Acta Ophthalmol (Copenh). 1972;50(3):375-84.

8. Kass MA, Sears ML. Hormonal regulation of intraocular pressure. Surv Ophthalmol. 1977;22(3):153-76.

9. Weinreb RN, Lu A, Beeson C. Maternal corneal thickness during pregnancy. Am J Ophthalmol. 1988;105(3):258-60.

10. Paterson GD, Miller SJ. Hormonal influence in simple glaucoma. A preliminary report. Br J Ophthalmol. 1963;47:129-37.

11. Horven I, Gjonnaes H, Kroese A. Blood circulating changes in the eye and limbs with relation to pregnancy and female sex hormones. Acta Ophthalmol (Copenh). 1976;54(2 p):203-14.

12. Imre J. Pregnancy and the eye: their endocrinological relations. XV Concilium. Ophthalmol Egypt. 1937;3:213-26 
13. Millodot $\mathrm{M}$. The influence of pregnancy on the sensitivity of the cornea. $\mathrm{Br} \mathrm{J}$ Ophthalmol. 1977;61(10):646-9.

14. Riss B, Riss P. Corneal sensitivity in pregnancy. Ophthalmologica. 1981;183(2): 57-62.

15. Duncan TE. Krukenberg spindles in pregnancy. Arch Ophthalmol. 1974;91(5): 355-8.

16. Talley AR, Assil KK, Schanzlin D. Patient selection and evaluation. In: Talamo JH, Krueger RR, editors. The excimer manual. Boston: Little, Brown \& Company; 1997. p.38.

17. Park SB, Lindahl KJ, Temnycky GO, Aquavella JV. The effect of pregnancy on corneal curvature. CLAO J. 1992;18(4):256-9.

18. Klein BE, Moss SE, Klein R. Effect of pregnancy on progression of diabetic retinopathy. Diabetes Care. 1990;13(1):34-40.

19. Chew EY, Mills JL, Metzger BE, Remaley NA, Jovanovic-Peterson L, Knopp RH, et al. Metabolic control and progression of retinopathy. The Diabetes in Early Pregnancy Study. National Institute of Child Health and Human Development Diabetes in Early Pregnancy Study. Diabetes Care. 1995;18(5):631-7.

20. Rosenn B, Miodovnik M, Kranias G, Khoury J, Combs CA, Mimouni F, et al. Progression of diabetic retinopathy in pregnancy: association with hypertension in pregnancy. Am J Obstet Gynecol. 1992;166(4):1214-8.

21. Akar Y, Yucel I, Akar ME, Zorlu G, Ari ES. Effect of pregnancy on intraobserver and intertechnique agreement in intraocular pressure measurements. Ophthalmologica. 2005;219(1):36-42.

22. Loukovaara S, Harju M, Kaaja R, Immonen I. Retinal capillary blood flow in diabetic and nondiabetic women during pregnancy and postpartum period. Invest Ophthalmol Vis Sci. 2003;44(4):1486-91.

23. Moloney JB, Drury MI. The effect of pregnancy on the natural course of diabetic retinopathy. Am J Ophthalmol. 1982;93(6):745-56.

24. Price JH, Hadden DR, Archer DB, Harley JM. Diabetic retinopathy in pregnancy. Br J Obstet Gynaecol. 1984;91(1):11-7.

25. Klein BE, Klein R, Meuer SM, Moss SE, Dalton DD. Does the severity of diabetic retinopathy predict pregnancy outcome? J Diabet Complications. 1988; 2(4):179-84.

26. Hemachandra A, Ellis D, Lloyd CE, Orchard TJ. The influence of pregnancy on IDDM complications. Diabetes Care. 1995;18(7):950-4.

27. Kaaja R, Sjöberg L, Hellsted T, Immonen I, Sane T, Teramo K. Long-term effects of pregnancy on diabetic complications. Diabet Med. 1996;13(2):165-9.

28. Chaturvedi N, Stephenson JM, Fuller JH. The relationship between pregnancy and long-term maternal complications in the EURODIAB IDDM Complications Study. Diabet Med. 1995;12(6):494-9.

29. Lövestam-Adrian M, Agardh CD, Aberg A, Agardh E. Pre-eclampsia is a potent risk factor for deterioration of retinopathy during pregnancy in Type 1 diabetic patients. Diabet Med. 1997;14(12):1059-65.

30. Axer-Siegel R, Hod M, Fink-Cohen S, Kramer M, Weinberger D, Schindel B, Yassur Y. Diabetic retinopathy during pregnancy. Ophthalmology. 1996;103(11): 1815-9.

31. Lauszus F, Klebe JG, Bek T. Diabetic retinopathy in pregnancy during tight metabolic control. Acta Obstet Gynecol Scand. 2000;79(5):367-70.

32. Chan WC, Lim LT, Quinn MJ, Knox FA, McCance D, Best RM. Management and outcome of sight-threatening diabetic retinopathy in pregnancy. Eye. 2004; 18(8):826-32.

33. Kitzmiller JL, Gavin LA, Gin GD, Jovanovic-Peterson L, Main EK, Zigrang WD. Preconception care of diabetes. Glycemic control prevents congenital anomalies. JAMA. 1991;265(6):731-6.

34. Rosenn B, Miodovnik M, Combs CA, Khoury J, Siddiqi TA. Glycemic thresholds for spontaneous abortion and congenital malformations in insulin-dependent diabetes mellitus. Obstet Gynecol. 1994;84(4):515-20.

35. The effect of intensive treatment of diabetes on the development and progression of long-term complications in insulin-dependent diabetes mellitus. The Diabetes Control and Complications Trial Research Group. N Engl J Med. 1993;329(14): 977-86.

36. Temple RC, Aldridge VA, Sampson MJ, Greenwood RH, Heyburn PJ, Glenn A Impact of pregnancy on the progression of diabetic retinopathy in Type 1 diabetes. Diabet Med. 2001;18(7):573-7.

37. Schultz KL, Birnbaum AD, Goldstein DA. Ocular disease in pregnancy. Curr Opin Ophthalmol. 2005;16(5):308-14. Review.

38. Chen YJ, Kuo HK, Huang HW. Retinal outcomes in proliferative diabetic retinopathy presenting during and after pregnancy. Chang Gung Med J. 2004;27(9): 678-84.

39. Jovanovic R, Jovanovic L. Obstetric management when normoglycemia is maintained in diabetic pregnan women with vascular compromise. Am J Obstet Gynecol. 1984;149(6):617-23.
40. Rabiah PK, Vitale AT. Noninfectious uveitis and pregnancy. Am J Ophthalmol. 2003;136(1):91-8

41. Ono K, Kunimatsu S, Numaga J, Kaburaki T, Fujino Y, Kawashima H. Multifocal choroiditis with subretinal fibrosis before, during and after pregnancy. Clin Experiment Ophthalmol. 2003;31(4):365-6.

42. Musiej-Nowakowska E, Ploski R. Pregnancy and early onset pauciarticular juvenile chronic arthritis. Ann Rheum Dis. 1999;58(8):475-80.

43. Chan CC, Reed GF, Kim Y, Agrón E, Buggage RR. A correlation of pregnancy term, disease activity, serum female hormones, and cytokines in uveitis. $\mathrm{Br} \mathrm{J}$ Ophthalmol. 2004;88(12):1506-9.

44. Perkins ES. Ocular toxoplasmosis. Br J Ophthalmol. 1973;57(1):1-17. Review.

45. Oniki S. Prognosis of pregnancy in patients with toxoplasmic retinochoroiditis. Jpn J Ophthalmol. 1983;27(1):166-74.

46. Amino N, Tanizawa O, Mori H, Iwatani Y, Yamada T, Kurachi K, et al. Aggravation of thyrotoxicosis in early pregnancy and after delivery in Graves' disease. J Clin Endocrinol Metab. 1982;55(1):108-12.

47. Neri A, Grausbord R, Kremer I, Ovadia J, Treister G. The management of labor in high myopic patients. Eur J Obstet Gynecol Reprod Biol. 1985;19(5):277-9.

48. Mayock RL, Sullivan RD, Greening RR, Jones R Jr. Sarcoidosis and pregnancy. J Am Med Assoc. 1957;164(2):158-63.

49. Hyman BN. Postpartum uveitis. Ann Ophthalmol. 1976;8(6):677-80.

50. Hallum AV. Eye changes in hypertensive toxemia of pregnancy: a study of three hundred cases. JAMA. 1936;106:1649-51.

51. Jaffe G, Schatz H. Ocular manifestations of preeclampsia. Am J Ophthalmol. 1987;103(3 Pt 1):309-15.

52. Watson DL, Sibai BM, Shaver DC, Dacus JV, Anderson GD. Late postpartum eclampsia: an update. South Med J. 1983;76(12):1487-9.

53. Fry WE. Extensive bilateral retinal detachment in eclampsia, with complete reattachment. Arch Ophthalmol. 1929;1:609-14.

54. Wagener HP. Lesions of the optic nerve and retina in pregnancy. JAMA. 1934; 103:1910-3.

55. Saito Y, Tano Y. Retinal pigment epithelial lesions associated with choroidal ischemia in preeclampsia. Retina. 1998; 18(2):103-8. Comment in: Retina. 1999; 19(3):262-3.

56. Cunningham FG, Fernandez CO, Hernandez C. Blindness associated with preeclampsia and eclampsia. Am J Obstet Gynecol. 1995;172(4 Pt 1):1291-8. Review.

57. Rahman J, Rahman W. Temporary blindness as a complication of eclampsia: observations on three cases. J Obstet Gynaecol. 2002;22(1):87-8.

58. Torres PJ, Antolin E, Gratacós E, Chamorro A, Cararach V. Cortical blindness in preeclampsia: diagnostic evaluation by transcranial Doppler and magnetic resonance imaging techniques. Acta Obstet Gynecol Scand. 1995; 74(8):642-4

59. Hiller KM, Honigman B. Cortical blindness in preeclampsia. Am J Emerg Med. 2004;22(7):631-2.

60. Na SJ, Hong JM, Park JH, Chung TS, Lee KY. A case of reversible postpartum cytotoxic edema in preeclampsia. J Neurol Sci. 2004;221(1-2):83-7.

61. Schaefer PW, Buonanno FS, Gonzalez RG, Schwamm LH. Diffusion-weighted imaging discriminates between cytotoxic and vasogenic edema in a patient with eclampsia. Stroke. 1997;28(5):1082-5.

62. Wilson SJ, Best RM, Love M, Kamel H. Cortical blindness following preeclampsia. Eye. 2000;14(Pt 2):254-6.

63. Monteiro ML, Hoyt WF, Imes RK. Puerperal cerebral blindness. Transient bilateral occipital involvement from presumed cerebral venous thrombosis. Arch Neurol. 1984;41(12):1300-1.

64. Beal MF, Chapman PH. Cortical blindness and homonymous hemianopia in the postpartum period. JAMA. 1980;244(18):2085-7.

65. Chumbley LC, Frank RN. Central serous retinopathy and pregnancy. Am J Ophthalmol. 1974;77(2):158-60.

66. Bedrossian RH. Letter: Central serous retinopathy and pregnancy. Am J Ophthalmol. 1974;78(1):152

67. Sunness JS, Haller JA, Fine SL. Central serous chorioretinopathy and pregnancy. Arch Ophthalmol. 1993;111(3):360-4.

68. Quillen DA, Gass DM, Brod RD, Gardner TW, Blankenship GW, Gottlieb JL. Central serous chorioretinopathy in women. Ophthalmology. 1996;103(1):72-9.

69. Seddon JM, MacLaughlin DT, Albert DM, Gragoudas ES, Ference M $3^{\text {rd }}$. Uveal melanomas presenting during pregnancy and the investigation of oestrogen receptors in melanomas. Br J Ophthalmol. 1982;66(11):695-704.

70. Siegel R, Ainslie WH. Malignant ocular melanoma during pregnancy. JAMA. 1963;185:542-3.

71. Chung CY, Kwok AK, Chung KL. Use of ophthalmic medications during pregnancy. Hong Kong Med J. 2004; 10(3):191-5. Comment in: Hong Kong Med J. 2004;10(5):365. 
72. Frishman WH, Chesner M. Beta-adrenergic blockers in pregnancy. Am Heart J. 1988;115(1 Pt 1):147-52. Review.

73. Lustgarten JS, Podos SM. Topical timolol and the nursing mother. Arch Ophthalmol. 1983;101(9):1381-2.

74. Ozawa H, Azuma E, Shindo K, Higashigawa M, Mukouhara R, Komada Y. Transient renal tubular acidosis in a neonate following transplacental acetazolamide. Eur J Pediatr. 2001;160(5):321-2.

75. Henkind P, Walsh JB, Berger AW. Physicians' Desk Reference for Ophthalmology. New York: Medical Economic; 1986.
76. De Santis M, Lucchese A, Carducci B, Cavaliere AF, De Santis L, Merola A, et al. Latanoprost exposure in pregnancy. Am J Ophthalmol. 2004;138(2): 305-6.

77. Kasirsky G, Lombardi L. Comparative teratogenic study of various corticoid ophthalmics. Toxicol Appl Pharmacol. 1970;16(3):773-8.

78. Shekleton P, Fidler J, Grimwade J. A case of benign intracranial hypertension in pregnancy. Br J Obstet Gynaecol. 1980;87(4):345-7.

79. Salamon SM. Tetracyclines in ophthalmology. Surv Ophthalmol. 1985;29(4): 265-75. Review. 